\title{
Research on Correction Coefficient of Damping Effect of Velocity Response Spectrum within the long and middle periods
}

\author{
Wang Mingzhen ${ }^{\mathrm{a}}$, Gao Lin ${ }^{\mathrm{b}}$ \\ College of Architecture Engineering, Chongqing University of Arts and Sciences, $319 \mathrm{Hong} \mathrm{He}$ \\ Street, Yong Chuan District, Chongqing 402160, China \\ awmz917@126.com, bgaolin32@163.com
}

Keywords: response spectrum; damping effect correction coefficient; characteristic period

\begin{abstract}
The seismic response analysis can be performed using the design speed response spectrum for the long and middle period structure. But the research results about the formula for calculating the damping effect correction coefficient of design velocity response spectrum in the long and middle period range is less. The classic calculation formulas of damping effect correction coefficients at home and abroad are compared firstly. The factors that affect the damping effect correction coefficient of response spectrum, the problems and deficiencies of the existing formulas are analyzed. And then the formula for calculating the damping effect correction coefficient of design velocity response spectrum in the long and middle period range is fitted based on the selected strong earthquake records.
\end{abstract}

\section{Introduction}

Damping ratio is the ratio of the viscous damping coefficient to the critical viscous damping factor of a vibration system. Damping ratio is a positive real number less than one. Different types of structures generally have different damping ratios in engineering practice. The damping ratio of steel structure is generally about $2 \% \sim 3 \%$. the damping ratio of reinforced concrete structure is generally about $5 \% \sim 7 \%$. The damping ratio of reinforced masonry structure is generally about $7 \% \sim 10 \%$. The equivalent damping ratio of energy dissipation structure with additional damper is about $10 \%$ 20\%, and the equivalent damping ratio of isolated structure can reach $20 \% \sim 30 \%{ }^{[1]}$. In addition, the damping ratio of the same structure type structure will vary with different factors such as the structure height, the layout of the structure anti lateral force components, whether the structure enters the elastic-plastic state or $\operatorname{not}^{[2]}$.

According to the response spectrum theory, the damping ratio is one of the important factors affecting the response spectrum amplitude. In general, the response spectrum amplitude decreases as the damping ratio increases. Especially for the short period structure with small initial damping ratio, the small increase of the damping ratio will cause the response spectrum amplitude to decrease significantly ${ }^{[3]}$. The variation regularity of design acceleration response spectrum, design velocity response spectrum and design displacement response spectrum when the damping ratio changes from $5 \%$ to $10 \%$ are shown in Fig.1.

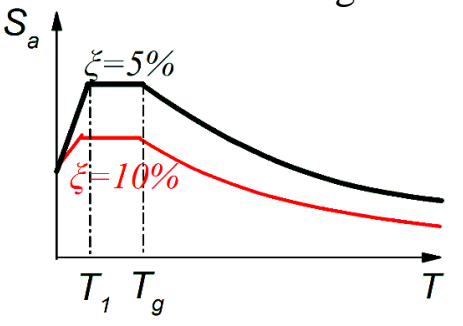

(a) design acceleration $\mathrm{RS}$

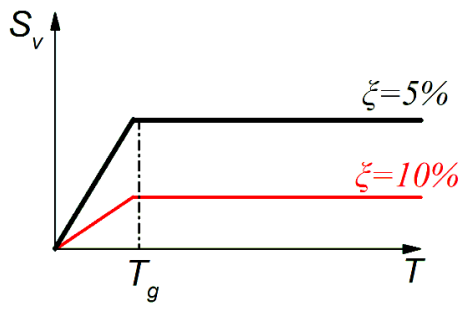

(b) design velocity $\mathrm{RS}$

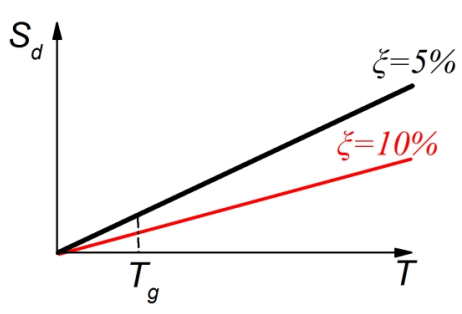

(c) design displacement RS

Fig. 1 The damping effect on design response spectrum

As can be seen from Fig.1, the amplitudes of three design response spectra have obvious changes when the damping ratio changes. For the design acceleration response spectrum, the short period range from $T_{l}$ to $T_{g}$ is the platform segments. The amplitude of design acceleration response spectrum does 
not change with structural period, and only changes with the damping ratio. For the design velocity response spectrum, the long period range greater than $T_{g}$ is the platform segments. The amplitude of design velocity response spectrum does not change with structural period, and only changes with the damping ratio. The characteristics of design displacement response spectrum changes with the damping ratio are shown in Fig.1(c).

Under normal circumstances, the reinforced concrete structures with more than 15 layers can be considered to belong to the long and middle period structure with structural period greater than $T_{g}$. For such structures, the design velocity response spectrum shown in Fig.1(b) should be used in structural response analysis using response spectrum theory.

For the design velocity response spectrum as shown in Fig.1(b), the influence of the damping ratio change on the response spectrum value when $T$ is greater than $T_{g}$ can be described by the damping correction coefficient of design velocity response spectrum with the long and middle period range as shown in Eq.1.

$$
D_{\xi}=\frac{S_{v}\left(T, \xi_{2}\right)}{S_{v}\left(T, \xi_{1}\right)}
$$

As can be seen from Fig.1(b), eq.1 is only related to the parameters of $\xi_{2}$ and $\xi_{1}$, and has nothing to do with the $T$ parameter.

The parameter $D_{\xi}$ plays an important role in the seismic design of building structures especially shock absorption structures. Since the seismic design response spectrum provided in the seismic design code is generally a design acceleration response spectrum with an initial damping ratio of $5 \%$, the damping effect correction coefficient $D_{\xi}$ should be used to estimate design response spectrum values when the damping ratio of designed structure is not equal to $5 \%$.

\section{Review of Classic Formulas for Damping Effect Correction Coefficient}

Since the 1950s, many experts and scholars in the world have begun to fully research the calculating formula of the damping effect correction coefficient for seismic response spectrum. The existing classic calculation formulas of damping effect correction coefficient for response spectrum are given in Table 1 .

Table 1 The calculation formulas of damping effect correction coefficient

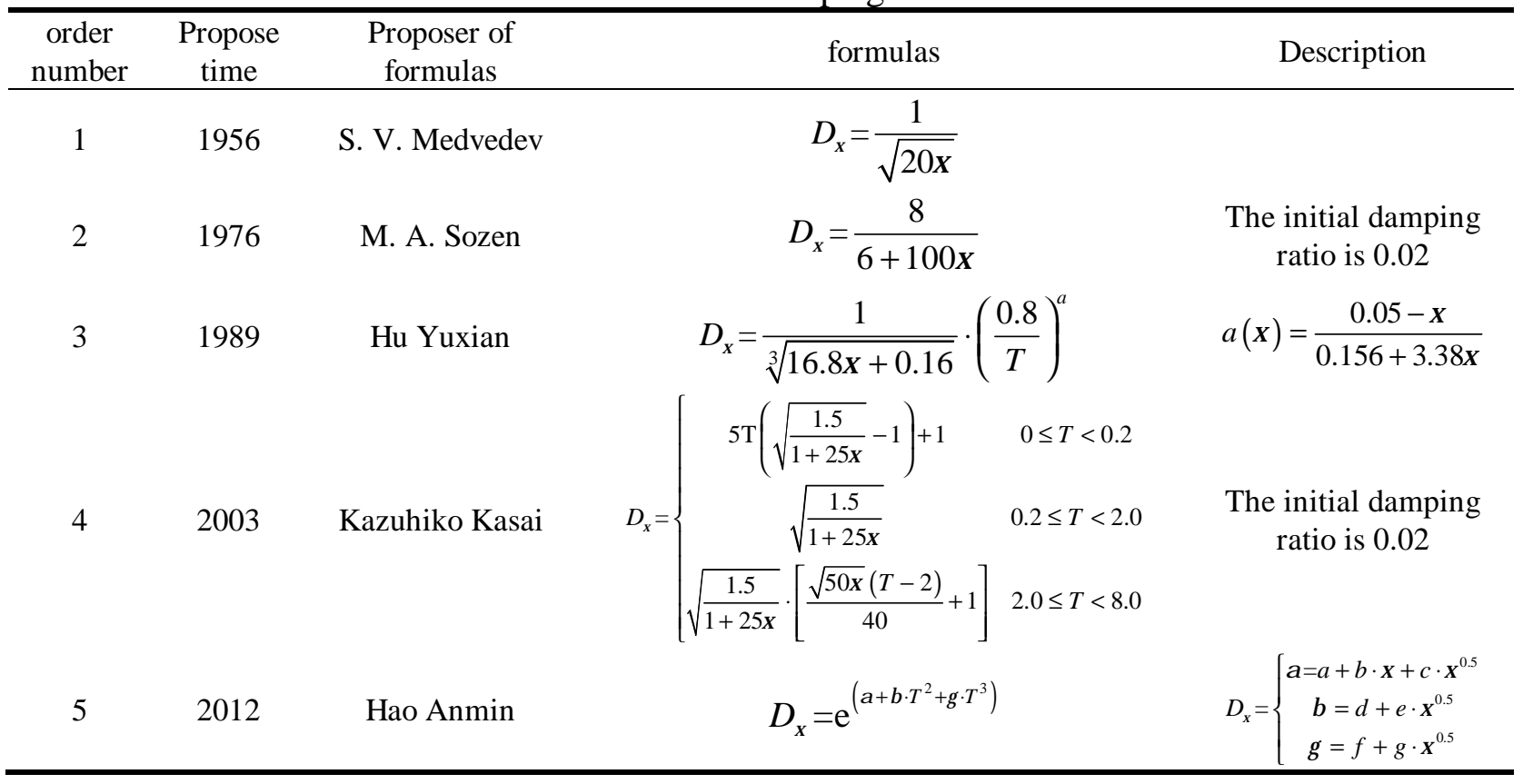

Among the formulas listed in the Table 1, the formula corresponding to number 4 is used to estimate the damping effect correction coefficient for velocity response spectrum. The formula corresponding 
to number 5 is used to estimate the damping effect correction coefficient for displacement response spectrum. Other formulas are used to estimate the damping effect correction coefficient for acceleration response spectrum.

In this paper, the main purpose of using the damping effect correction coefficient is to evaluate the degree of amplitude variation for velocity response spectrum in the long and middle period range. There are only a few formulas for calculating the damping effect correction coefficient of velocity response spectrum in the current professional field, and there are two major deficiencies existing in the current formulas. Some formulas only consider the influence of the damping ratio and do not consider the influence of other factors on the formula. Although some formulas take into account the factors of site classification, damping ratio and others on the formulas, but the formulas are complex and inconvenience, which some parameters need to lookup table to determine. Therefore, it is necessary to further study the formula for calculating the damping effect correction coefficient of velocity response spectrum in the long and middle period range.

\section{Influencing factors of Damping Effect Correction Coefficient}

According to the classic formulas related to the damping effect correction coefficients in Table 1, most of the formulas only consider the damping ratio influence on the damping effect correction coefficients. In fact, the damping effect correction coefficients are also related to the factors of site classification, ground motion properties and so on.

Based on comprehensive analyses of the characteristics and influencing factors for the existing calculation formulas of damping effect correction coefficients at home and abroad, the damping ratio and characteristic period are mainly considered when the new formula of damping effect correction coefficient are fitted in this paper. In addition, the fitting formula of damping effect correction coefficient in this paper is used to calculate the variation amplitude of velocity response spectrum in the long and middle period range when the damping ratio changes. The characteristic period can be calculated by Eq.2 and Eq.3.

$$
T_{g}=2 \pi \frac{E P V}{E P A}
$$

In Eq.2, $E P V$ is the effective peak velocity and $E P A$ is the effective peak acceleration. The calculation of $E P V$ and $E P A$ are shown in Eq.3.

$$
E P V=\frac{\bar{S}_{v}}{2.5}, E P A=\frac{\bar{S}_{a}}{2.5}
$$

In Eq.3, the parameter $\bar{S}_{a}$ is the average value of the amplitude for acceleration response spectrum in the range of 0.1 second to 0.5 second with the damping ratio of $5 \%$. And the parameter $\overline{S_{v}}$ is the average value of the amplitude for velocity response spectrum in the range of 0.8 second to 1.2 second with the damping ratio of $5 \%{ }^{[4]}$. The number 2.5 in the Eq. 3 is an empirical coefficient.

\section{Formula fitting of Damping Effect Correction Coefficient}

In order to fit the calculation formula of damping effect correction coefficient, the strong earthquake records are selected from Japan earthquake observation network named Kik-net, which are 3.11 Japan earthquake records from 40 strong earthquake stations.

Based on the collected strong earthquake records, the following steps are used to fit the correction formula of damping effect correction coefficient for velocity response spectrum in the long and middle period range.

First of all, MATLAB software is used to program to calculate the 40 records response spectrum and estimate the epicentral distances corresponding to every earthquake records. The 720 sets data including acceleration response spectra and velocity response spectra with the damping ratio of $0,0.02$, $0.05,0.10,0.15,0.20,0.25,0.3$ and 0.4 respectively. Then, the characteristic period of each seismic 
record is calculated according to Eq.2 and Eq.3. Finally, the automatic searching and fitting function of the "common global optimization algorithm" and "Levenberg-Marquardt" optimization algorithm that comes with the 1stopt software is used. The formula parameters and the final expression after fitting are shown in Eq.4.

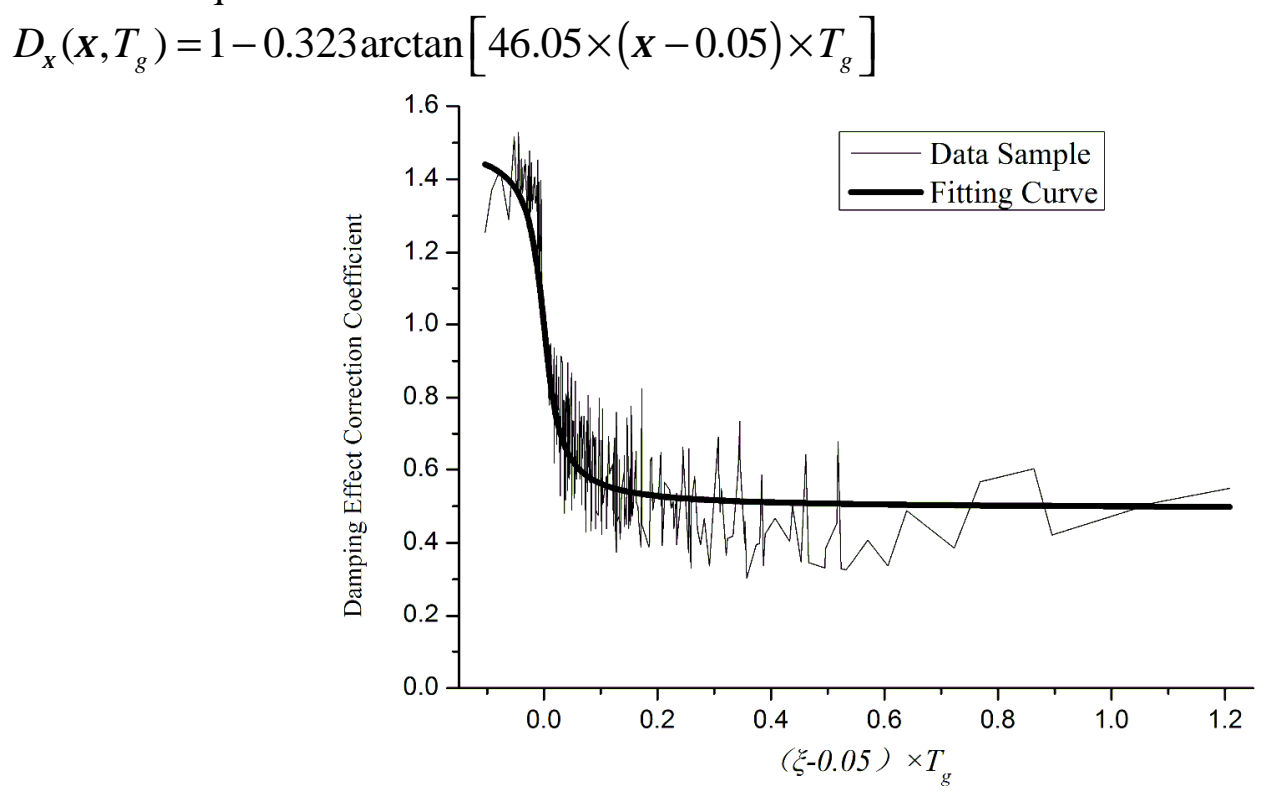

Figure 2 The correspondence between the theoretical calculated values and the measured value

For the fitting Eq.4, the correlation coefficient $R$ is 0.939, the mean square deviation $D$ is 0.107 .

From the statistical index obtained from the fitting results and the formula basic form, it can be seen that the Eq.4 have the features of easy operation, simple form and high accuracy. The correspondence between the theoretical calculated values calculated using Eq.4 and the measured value of the sample is plotted in Fig.2.

The using conditions of the fitting formula in this paper for calculating the damping effect correction coefficient of velocity response spectrum should be paid attention.

First of all, the Eq.4 fitted in this paper is suitable for the calculation of damping effect influence coefficient for velocity response spectrum in the long and middle period range. That is, it can only be directly applied to estimate and calculate the amplitude changes of velocity response spectrum when the damping ratio changes from 0.05 to other values.

Secondly, since only the data samples with damping ratios varying from 0.02 to 0.4 are selected for fitting formula, the damping ratio should be chosen between 0.02 and 0.4 when using Eq.4. That is, Eq. 4 is not suitable for evaluating the amplitude variation of velocity response spectrum in the long and middle period range when the damping ratio changes outside the interval from 0.02 to 0.4 . It can be said that the applicable range of the damping ratio does not have the extensibility.

\section{Conclusions}

To sum up, the following three aspects contents have been completed.

1) The existing formulas for calculating damping effect influence coefficient of response spectra are summed up. The characteristics, applicable ranges and limitations of the existing formulas are analyzed. The need to fit the formula for calculating the damping effect influence coefficient of velocity response spectrum in the long and middle period range is described.

2) A certain number of stations and ground motion data with more complete information are selected according to certain principles. The site classification and PGA amplitude selection range of the stations are processed and analyzed. Which are subsequent preparations for fitting the formula for calculating the damping effect influence coefficient of velocity response spectrum. 
3) The calculation formula with the features of easy operation, simple form and high accuracy for damping effect correction coefficient of velocity response spectrum is fitted through using advanced optimization fitting software 1stopt.

\section{Acknowledgements}

This work was financially supported by the Scientific and Technological Research Program of Chongqing Municipal Education Commission (KJ1601132), the Scientific Research Fund for Chongqing University of Arts and Sciences (2017RJJ32, R2015JJ06) and National Natural Science Foundation of China (51678544).

\section{References}

[1] L. A. Wyllie, and J. R. Filson. Performance of Engineered Structures, Earthquake Spectra, Special Supplement, Armenia Earthquake Reconnaissance Report, 1989,70-92.

[2] B. T. Sun, M. Z. Wang, L. Gao. Design principles for stiffness-tandem energy dissipation coupling beam, Smart Structures and Systems, 2017, 20(1): 53-60.

[3] H. B. You, F. X. Zhao, H. You. Characteristic period value of the seismic design response spectrum of UHV electrical equipment, Earthquake Research in China, 2015, 29 (1): 117-127.

[4] Y. F. Zhao, G. S. Tong. An investigation of characteristic periods of seismic ground motions, Journal of Earthquake Engineering, 2009, (13):540-565. 